\title{
Modulation of the balance of fatty acid production and secretion is crucial for enhancement of growth and productivity of the engineered mutant of the cyanobacterium Synechococcus elongatus
}

Akihiro Kato ${ }^{1}, K^{\prime}$ azuhide Use ${ }^{1}$, Nobuyuki Takatani ${ }^{1,4}$, Kazutaka Ikeda ${ }^{2,4,5}$, Miyuki Matsuura ${ }^{1}$, Kouji Kojima ${ }^{1,4}$, Makiko Aichi ${ }^{3,4}$, Shin-ichi Maeda ${ }^{1,4}$ and Tatsuo Omata ${ }^{1,4,6^{*}}$

\begin{abstract}
Background: Among the three model cyanobacterial species that have been used for engineering a system for photosynthetic production of free fatty acids (FFAs), Synechococcus elongatus PCC7942 has been the least successful; the FFA-excreting mutants constructed from this strain could attain lower rates of FFA excretion and lower final FFA concentrations than the mutants constructed from Synechocystis sp. PCC6803 and Synechococcus sp. PCC7002. It has been suggested that S. elongatus PCC7942 cells suffer from toxicity of FFA, but the cause of the low productivity has remained to be determined.

Results: By modulating the expression level of the acyl-acyl carrier protein thioesterase and raising the light intensity during cultivation, FFA secretion rates comparable to those obtained with the other cyanobacterial species were attained with an engineered Synechococcus elongatus mutant (dAS1T). The final FFA concentration in the external medium was also higher than previously reported for other S. elongatus mutants. However, about $85 \%$ of the total FFA in the culture was found to remain in the cells, causing severe photoinhibition. Targeted inactivation of the wzt gene in dAS1T, which gene manipulation was previously shown to result in loss of the hydrophilic O-antigen layer on the cell surface, increased FFA secretion, alleviated photoinhibition, and lead to 50 and $45 \%$ increase in the final cell density and the total amount of FFA in the culture (i.e., the sum of the cellular and extracellular FFA), respectively. The average rate of production of total FFA by the culture of the $\triangle w z t$ strain was $2.7 \mathrm{mg} \mathrm{L}^{-1} \mathrm{~h}^{-1}$, being five times higher than those reported for Synechocystis sp. PCC 6803 and comparable to the rates of triacylglycerol production in green algae.
\end{abstract}

Conclusion: Synechococcus elongatus PCC7942 has larger capacity of FFA production than Synechocystis sp. PCC6803 but accumulates most of the product in the cell because of the imbalance of the rates of FFA production and secretion. This causes severe photoinhibition and exerts adverse effects on cell growth and FFA productivity. Enhancement of FFA secretion would be required to fully exploiting the capacity of FFA production for the purpose of biofuel production.

Keywords: Cyanobacteria, Biofuel production, Free fatty acids, O-antigen

\footnotetext{
*Correspondence: omata@agr.nagoya-u.ac.jp

${ }^{6}$ Laboratory of Molecular Plant Physiology, Graduate School

of Bioagricultural Sciences, Nagoya University, Furo, Chikusa,

Nagoya 464-8601, Japan

Full list of author information is available at the end of the article
} 


\section{Background}

Production of free fatty acids (FFAs) using genetically engineered cyanobacteria is thought to be a promising method of production of renewable biofuels, because FFAs are excreted into the medium and as a result, the two energy-intensive steps in microbial biofuel production, i.e., recovery of the cells from the growth medium and extraction from the cells of the photosynthetic product, can be omitted [1-3]. The highest reported yield of FFA production to date is a final FFA concentration of $197 \mathrm{mg} \mathrm{L}^{-1}$ achieved with an average production rate of $0.438 \mathrm{mg} \mathrm{L}^{-1} \mathrm{~h}^{-1}$ obtained with an engineered strain of Synechocystis sp. PCC 6803 [1]. However, the yield of cyanobacteria-based FFA production is much lower than that reported for algae-based triacylglycerol (TAG) production [4].

FFA production can be achieved in cyanobacteria by two means: (1) inactivation of the endogenous gene encoding acyl-ACP synthetase (Aas) [5], which recycles FFA via esterification to ACP, and (2) introduction of a foreign thioesterase(s) having the capacity to hydrolyze acyl-acyl carrier protein (ACP) [1]. Aas-deficient mutants expressing truncated thioesterase from E. coli ('TesA) have been constructed from three strains of model cyanobacteria, Synechocystis sp. PCC6803, Synechococcus sp. PCC7002, and Synechococcus elongatus PCC7942. In Synechocystis sp. PCC6803, an extracellular FFA concentration of $83.6 \mathrm{mg} \mathrm{L}^{-1}$ with an average FFA excretion rate of $0.17 \mathrm{mg}^{-1} \mathrm{~L}^{-1} \mathrm{~h}^{-1}$ was attained by simply introducing 'tesA and inactivating aas, and the FFA productivity was further improved to attain the extracellular FFA level of $197 \mathrm{mg} \mathrm{L}^{-1}$ (see above) by additional gene manipulation(s) aimed at inactivation of the PHB biosynthesis pathway, weakening of the peptidoglycan layer, overexpression of acetyl-CoA carboxylase, and expression of thioesterases from various sources other than E. coli [1]. In Synechococcus sp. PCC 7002 and Synechococcus elongatus PCC 7942, the tesA-expressing aas-deficient mutants showed relatively low FFA productivity, attaining extracellular FFA concentrations of 40 and $49.3 \mathrm{mg} \mathrm{L}^{-1}$ with average excretion rates of 0.083 and $0.103 \mathrm{mg} \mathrm{L}^{-1} \mathrm{~h}^{-1}$, respectively [2, 3]. While FFA production by the Synechococcus sp. PCC7002 mutant was increased to $131 \mathrm{mg} \mathrm{L}^{-1}$ with an average excretion rate of $0.27 \mathrm{mg} \mathrm{L}^{-1} \mathrm{~h}^{-1}$ by overexpression of Rubisco [2], similar gene manipulation failed to increase FFA production by S. elongatus PCC7942 mutants, leading to a hypothesis that adverse effects of FFA on cellular activities are limiting FFA production in the latter strain [6]. Although the molecular basis of FFA toxicity remains to be determined, these results indicated that different factors limit FFA production in different cyanobacterial species [2].
In an attempt to increase FFA production per-cell of S. elongatus PCC7942 mutants, Kato et al. [7] overexpressed tesA while minimizing cell growth by nitrogen limitation. This, however, killed the cells, suggesting that overproduction of FFAs during growth limitation is lethal. A fortuitously obtained pseudorevertant capable of growth under these conditions was found to overexpress an RND-type efflux system having the capacity of FFA export and shown to excrete FFAs at an average rate of $0.23 \mathrm{mg} \mathrm{L}^{-1} \mathrm{~h}^{-1}$, which was twice as high as the rate that had been attained in S. elongatus PCC7942 [7]. The pseudorevertant nevertheless could not sustain growth for $>200 \mathrm{~h}$ and the final FFA concentration was only $45 \mathrm{mg} \mathrm{L}^{-1}$, being similar to the previously reported values. In this study, we show that modulation of the balance of FFA production in the cell and FFA export out of the cell is essential to further increase the FFA productivity. In spite of a high FFA excretion rate thus attained, which is similar to the highest rate ever reported in cyanobacterial mutants, the mutant cells are shown to retain most of the FFAs intracellularly and suffer from photoinhibition. Possible strategies to exploit the unexpectedly large capacity of FFA synthesis found in S. elongatus PCC7942 for biofuel production are discussed.

\section{Results}

\section{Improvement of FFA productivity by attenuation of 'tesA} expression

In the FFA-producing strain dAS1T, the 'tes $A$ coding sequence was transcriptionally fused to the nitrogenregulated promoter $\left(P_{\text {nirA }}\right)$ of the nitrate assimilation operon (nirA-nrtABCD-narB) and introduced into the aas locus (Fig. 1a, b). While $P_{\text {nirA }}$ is inactive in the presence of ammonium in growth media, it sustains low but robust expression of the downstream genes when the cells are grown in media containing sufficient amounts of nitrate as the nitrogen source $[8,9]$. Semi-quantitative RT-PCR analysis of the tes $A$ transcript confirmed the nitrogen-responsive regulation of the $P_{\text {nirA }}$ promoter in dAS1T (Fig. 1c). Since $P_{\text {nirA }}$ is most active under constantly nitrate-limited growth conditions, which can be achieved in a chemostat or by forcing nitrate transporterless mutants to grow slowly in media containing high concentrations of nitrate that allow for slow, passive diffusion of nitrate into the cell $[8,9]$, Kato et al. [7] once introduced the same $P_{\text {nirA }}$ - $^{\text {tes }} A$ transcriptional fusion to the aas locus in a nitrate transporter-less $S$. elongatus mutant to maximize FFA production while minimizing cell growth [7]. However, the resultant mutant (dAS2T) died under the 'tesA-inducing conditions in nitrate-containing media, showing that overexpression of 'tes $A$ is lethal. Unlike dAS2T, dAS1T could grow 

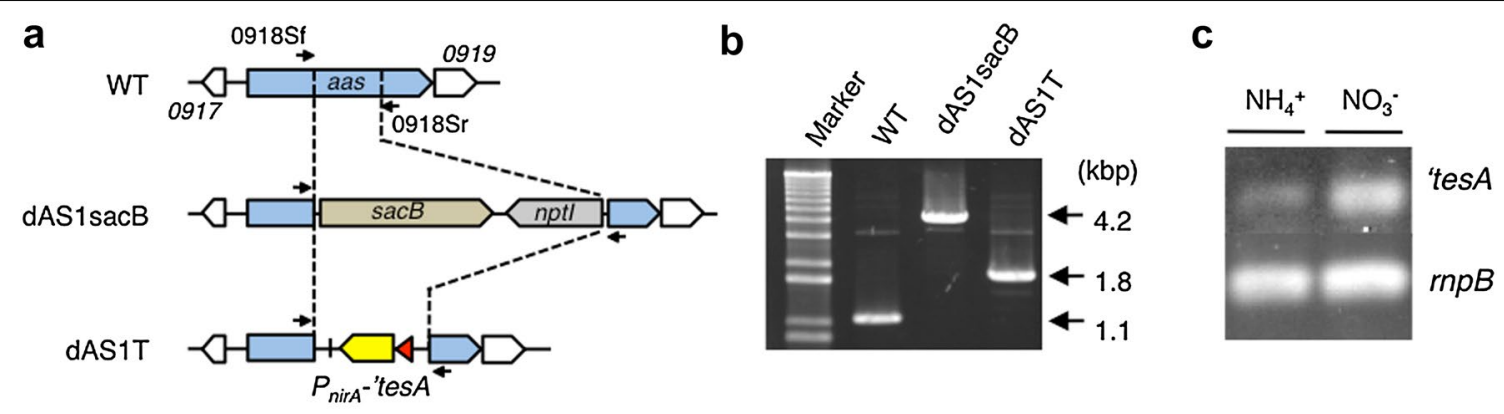

$1 \mathrm{kbp}$

Fig. 1 Construction of the FFA-producing strains from S. elongatus. a Map of the aas region of the genomes of WT and the mutants. A transcriptional fusion of the nirA promoter from S. elongatus (shown in red) and the 'tesA coding sequence from E. coli (shown in yellow) was used to replace an intragenic 662 bp fragment of the aas gene via the marker-exchange eviction method to construct dAS1T. Arrows above the map indicate the primers used for PCR analysis in $\mathbf{b}$. $\mathbf{b}$ PCR analysis of the aas region of WT and the mutants. c Semi-quantitative RT-PCR analysis of the 'tesA transcript in AAS1T, showing the effect of nitrogen source. Total RNA was extracted from dAS1T cells at $t=48 \mathrm{~h}$ and subjected to the analysis, using the $r n p B$ transcript as a control. Cycle numbers for PCR were 24 and 26 for'tes $A$ and $r n p B$, respectively

in the nitrate-containing medium, presumably due to attenuation of tesA expression (Fig. 2a). Under lowlight conditions $\left(50 \mu \mathrm{E} \mathrm{m}^{-2} \mathrm{~s}^{-1}\right)$ in the nitrate-containing medium, dAS1T secreted FFA at an average rate of $0.27 \mathrm{mg} \mathrm{L}^{-1} \mathrm{~h}^{-1}$ (Fig. 2a, filled circles), attaining a FFA concentration in the medium of $64 \mathrm{mg} \mathrm{L}^{-1}$ in $240 \mathrm{~h}$. These figures were higher than those obtained previously under similar growth conditions in a S. elongatus PCC 7942 aas mutant carrying a $P_{t r c}$-driven 'tes $A$ : an average excretion rate of $0.1 \mathrm{mg} \mathrm{L}^{-1} \mathrm{~h}^{-1}$ and a final FFA concentration of $49 \mathrm{mg} \mathrm{L}^{-1}$ obtained in $480 \mathrm{~h} \mathrm{[3].} \mathrm{These} \mathrm{results}$ seemed to suggest that attenuation of tes $A$ expression contributed to the improved FFA productivity of dAS1T.

\section{Enhanced FFA production under high-light conditions and its toxic effects}

The FFA secretion rate of dAS1T in the nitrate-containing medium was increased by raising the light intensity to $180 \mu \mathrm{E} \mathrm{m}^{-2} \mathrm{~s}^{-1}$ (Fig. 2a, open circles); the average FFA excretion rate of $0.35 \mathrm{mg} \mathrm{L}^{-1} \mathrm{~h}^{-1}$ was thus attained, but the cells died in $240 \mathrm{~h}$ of cultivation with complete loss of chlorophyll, showing blue coloration due to the remaining phycobiliproteins (Fig. 2b). When grown on ammonium under the high-light conditions, dAS1T showed slower excretion of FFA and remained green (Fig. 2a triangles and $b$ ). These results showed that the highlight condition per se was not toxic to the cells. Using an aas single mutant of $S$. elongatus (dAS1), Takatani et al. [10] showed that production of FFAs renders the cyanobacterial cells sensitive to photodamage [10]. Since dAS1 did not show such a severe growth phenotype as was observed with dAS1T [10], it was deduced that the increased FFA production due to expression of 'TesA

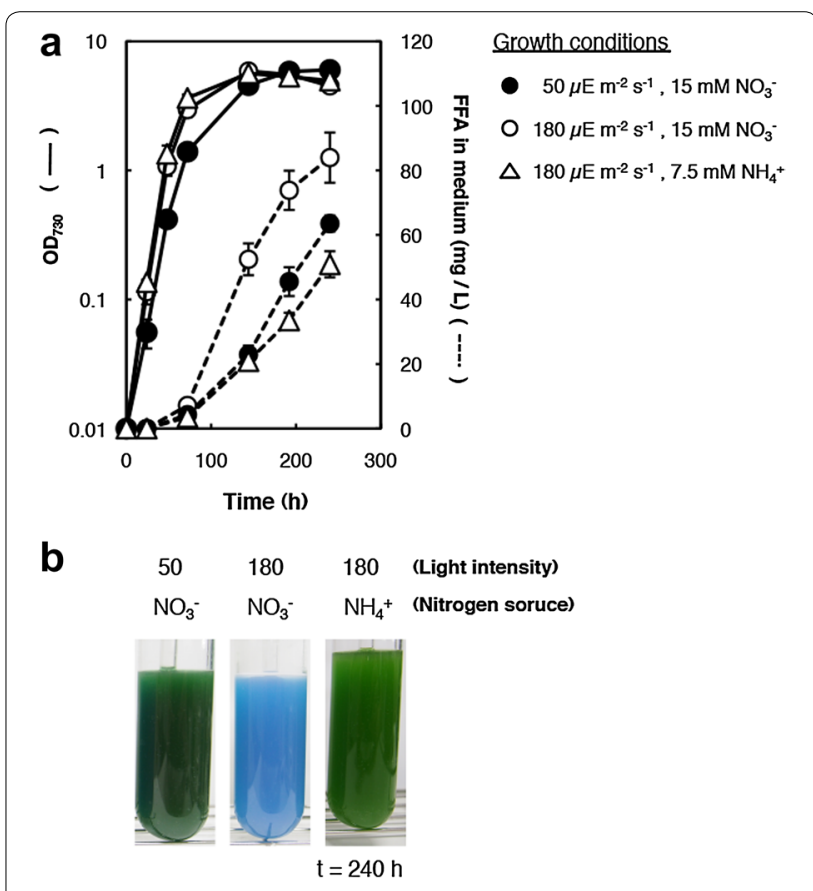

Fig. 2 Effects of light intensity and nitrogen source on growth and FFA production. a Growth and FFA production of dAS1T. Cells grown in nitrate-containing medium under low light $\left(50 \mu \mathrm{E} \mathrm{m}^{-2} \mathrm{~s}^{-1}\right)$ were harvested and transferred to nitrate or ammonium-containing

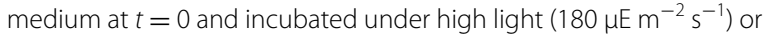
low light $\left(50 \mu \mathrm{E} \mathrm{m}^{-2} \mathrm{~s}^{-1}\right)$. Solid lines optical density at $730 \mathrm{~nm}\left(\mathrm{OD}_{730}\right)$; dotted lines FFA concentrations in medium. Closed circles cells grown in nitrate-containing medium under low light $(n=3)$; Open circles cells grown in nitrate-containing medium under high light $(n=6)$; Open triangles cells grown in ammonium-containing medium under high light $(n=3)$. Data shown are the mean \pm SE from the biological replicates. b Appearance of the cultures at $t=240 \mathrm{~h}$, showing the growth defect of dAS1T in nitrate-containing medium under high light after prolonged cultivation 
lead to the growth inhibition in dAS1T under the highlight conditions.

\section{Toxicity of FFA overproduction is alleviated by impairment} of O-antigen production

The adverse effects of enhanced FFA production on growth of the dAS1T cells suggested that intracellular accumulation of FFA was toxic. To further improve the FFA productivity of dAS1T, we attempted to facilitate passive diffusion of FFAs out of the cells by modification of the cell surface structure. Cyanobacteria as well as other gram-negative bacteria have inner (cytoplasmic) and outer membranes, with lipopolysaccharide (LPS) being a major component of the latter. The polysaccharide portion of LPS, consisting of the $\mathrm{O}$-antigen chain and the core oligosaccharide complex, comprises the hydrophilic layer on the cell surface, which has been shown to act as a diffusion barrier to protect bacterial cells against extracellularly added FFAs [11, 12]. This suggested that elimination of O-antigen, which comprises the outermost and the largest portion of the polysaccharide chain of LPS, might help release of FFAs out of the FFA-producing cyanobacterial cells. We hence constructed in this study an $\mathrm{O}$-antigen deficient mutant from dAS1T by inactivating the $w z t$ gene involved in the export of the $\mathrm{O}$-antigen unit across the cytoplasmic membrane [13]. Impairment of O-antigen production was reported to render the cell surface of S. elongatus hydrophobic and to confer the cells the resistance against grazing by amoebae, without exerting any negative effects on growth [13]. The gene was successfully inactivated (Fig. 3) and the resulting mutant showed the autoflocculation phenotype as previously reported [13].

Figure 4Aa compares growth of dAS1T and dAS1T $\Delta w z t$ strains with that of WT under the high-light conditions with nitrate as the nitrogen source. Growth of dAS1T was much slower than that of WT, with the final cell density being about $35 \%$ of the WT level. The
$\mathrm{dAS1T} \Delta w z t$ strain grew faster than the parental dAS1T strain and attained a final cell density corresponding to about $50 \%$ of WT. Unlike the dAS1T strain, which completely lost chlorophyll in $240 \mathrm{~h}$, the dAS1T $\Delta w z t$ cells retained chlorophyll after $240 \mathrm{~h}$ of cultivation (Fig. 4Ab). Thus, the loss of $\mathrm{O}$-antigen alleviated the toxic effects exerted by FFA production under the high-light conditions. It should be noted, however, that the $\mathrm{dAS1T} \Delta w z t$ cultures turned into blue in coloration in $264 \mathrm{~h}$ (not shown), indicating that the "early-death" phenotype of the dAS1T strain was partially rescued by the loss of O-antigen.

Measurements of FFA concentration in the medium during cultivation of the FFA-producing strains revealed a small increase in the rate of FFA excretion in the $\mathrm{dAS1T} \Delta w z t$ strain compared with the parental dAS1T strain (Fig. 4Ba). The average rate of FFA excretion during the $240 \mathrm{~h}$ of cultivation was $0.41 \mathrm{mg} \mathrm{L}^{-1} \mathrm{~h}^{-1}$ in $\mathrm{dAS1T} \Delta w z t$, which was about $15 \%$ higher than that in $\mathrm{dAS1T}$ and close to the highest rate achieved in cyanobacteria: $0.438 \mathrm{mg} \mathrm{L}^{-1} \mathrm{~h}^{-1}$ obtained with an engineered strain of Synechocystis sp. PCC 6803 [1]. The FFA concentration in the external medium of dAS1T $\Delta w z t$ reached $100 \mathrm{mg} \mathrm{L}^{-1}$, which was $12 \%$ higher than that attained by dAS1T.

\section{O-antigen deficiency results in improvement of FFA productivity}

In both dAS1T and dAS1T $\Delta w z t$, the cellular FFA content was initially low and was increased during growth (Fig. 4Bb). In the initial phase of growth (up to $t=72 \mathrm{~h}$ ), cellular FFA increased in a similar manner in dAS1T and $\mathrm{dAS1T} \Delta w z t$, but the increase was slower thereafter in $\mathrm{dAS} 1 \mathrm{~T} \Delta w z t$ than in dAS1T. At $t=144 \mathrm{~h}$, cellular contents of FFAs were calculated to be 175 and $155 \mathrm{mg}$ per $\mathrm{mL}$ of cell volume for dAS1T and dAS1T $\Delta w z t$, respectively. The total amount of FFAs in the culture, as calculated from the data shown in Fig. 4Aa, Ba, b, increased in
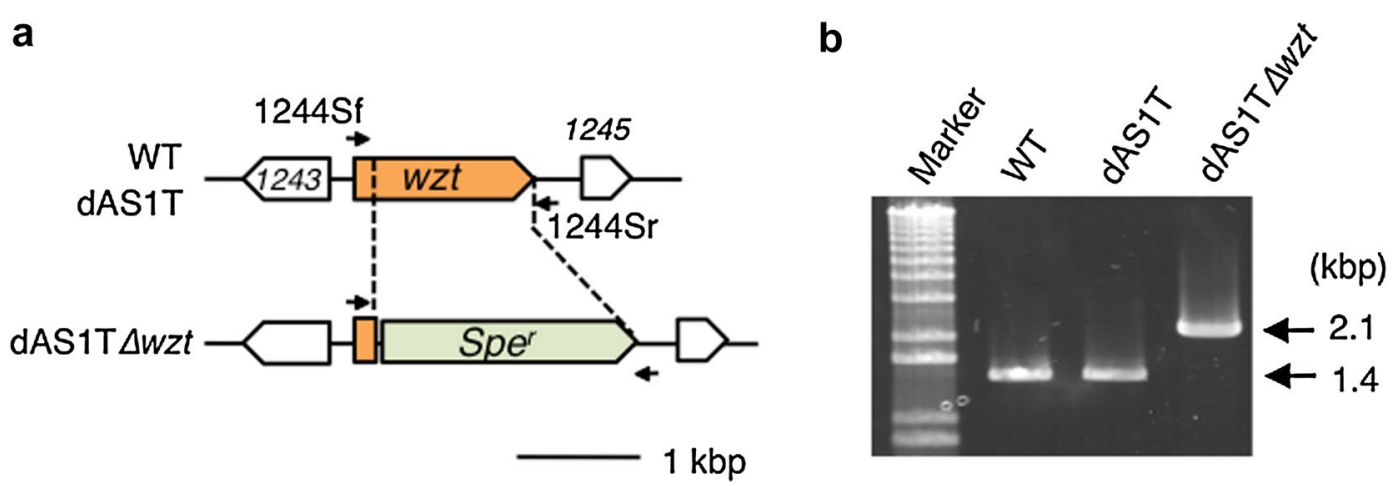

Fig. 3 Construction of the wzt-deficient mutant from dAS1T. a Map of the wzt region of the genomes of WT, dAS1T, and dAS1T $\triangle W Z t$. Arrows above the map indicate the primers used for PCR analysis in $\mathbf{b} . \mathbf{b}$ PCR analysis of the wzt region of the genomes of WT, dAS1T, and dAS1T $\triangle W Z t$ 
A

a $\left(\times 10^{9}\right)$

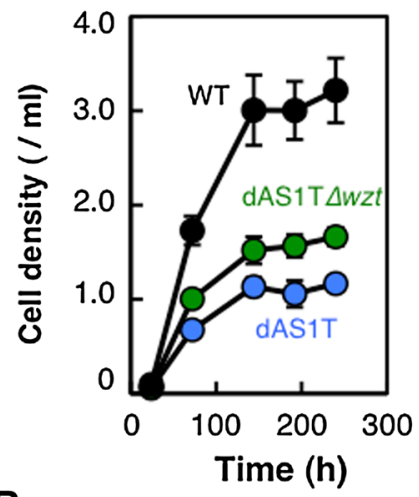

b

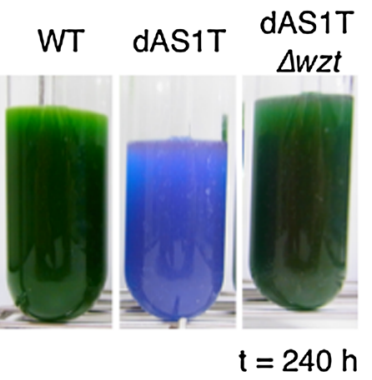

B

b
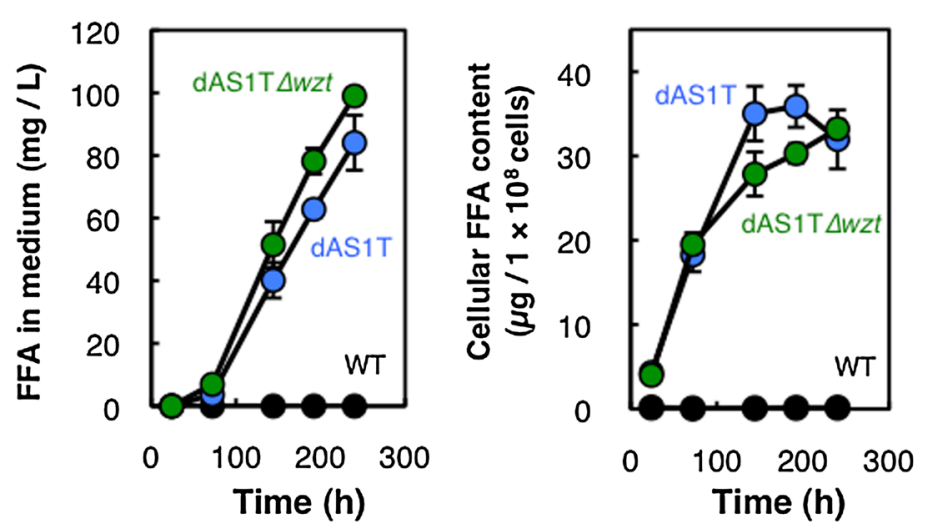

C

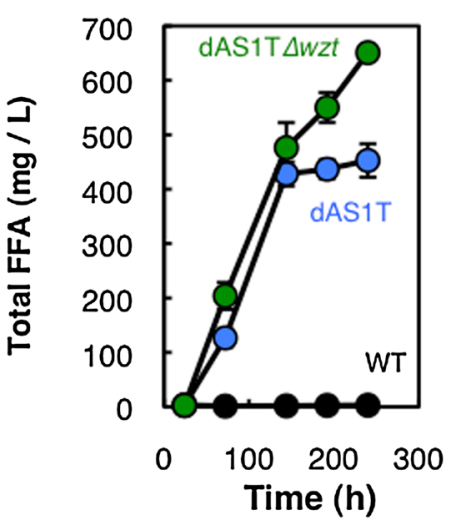

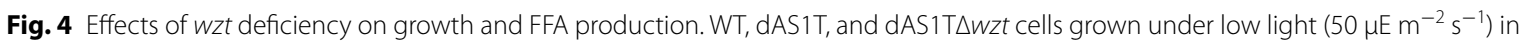
nitrate-containing medium were inoculated at time zero into new nitrate-containing medium and incubated under the high-light conditions $\left(180 \mu \mathrm{E} \mathrm{m}^{-2} \mathrm{~s}^{-1}\right)$. A Cell density measured at designated times (a) and appearance of the cultures at $t=240 \mathrm{~h}(b)$. B Changes in extracellular FFA concentration (a), cellular FFA content (b), and the sum of cellular and extracellular FFA in the cultures (c). Black circles WT; blue circles dAS1T; green circles dAS1T $\Delta w z t$. Data shown are the mean \pm SE from biological triplicates

a similar manner in the two strains until they reached the stationary phase of growth at $t=144 \mathrm{~h}$ (Fig. 4Bc). During the stationary phase, there was essentially no increase in total FFAs in the dAS1T culture. The continued increase of extracellular FFAs (Fig. 4Ba) and a decline in cellular FFA content (Fig. 4Bb) in the dAS1T cultures during the stationary phase suggested that FFAs are released from dead cells in the last stage of cultivation. Unlike dAS1T cells, the cells of dAS1T $\Delta w z t$ continued FFA production during the stationary phase to attain the total FFA level of $650 \mathrm{mg} \mathrm{L}^{-1}$, which was about $45 \%$ higher than that in the dAS1T cultures. The average rate of production of total FFA by the dAS1T $\Delta w z t$ culture was $2.7 \mathrm{mg} \mathrm{L}^{-1} \mathrm{~h}^{-1}$, being comparable to some of the examples of the rate of TAG production by green algae [14]. Although dAS1T $\Delta w z t$ attained the highest level of extracellular FFA ever reported for the FFA-producing strains obtained from $S$. elongatus [3], it should be noted that most of FFA in the culture was retained in the cell.

In $\mathrm{dAS} 1 \mathrm{~T} \Delta w z t$ as well as in $\mathrm{dAS} 1 \mathrm{~T}$, palmitic acid (16:0) was the major constituent of both the cellular and extracellular pools of FFAs, comprising $>60 \%$ of the total FFAs (Fig. 5). Together with stearic acid (18:0) and myristic acid (14:0), the saturated fatty acids comprised about $90 \%$ and $85 \%$ of the cellular and extracellular FFA pools, respectively. As previously observed [5, 7], the relative contents of palmitoleic acid (16:1) and myristoleic acid (14:1) were significantly higher in the extracellular FFA pool than in the cellular pool in both of the strains (Fig. 5). There was, however, no significant difference between the dAS1T and dAS1T $\Delta w z t$ strains in their cellular and extracellular FFA profiles. 


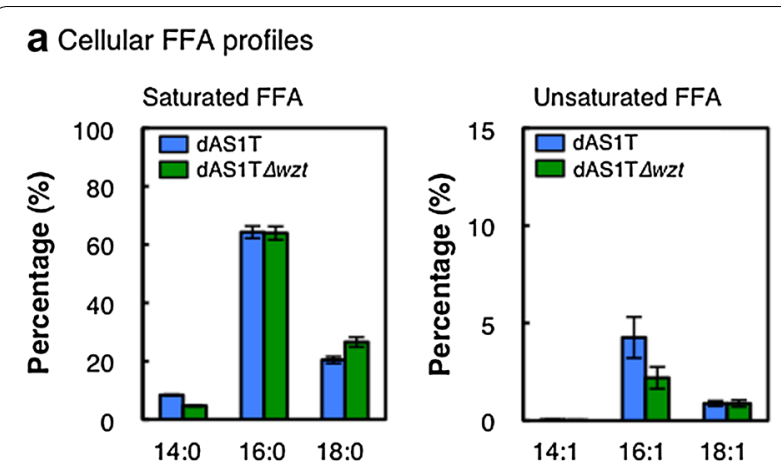

b Extracellular FFA profiles
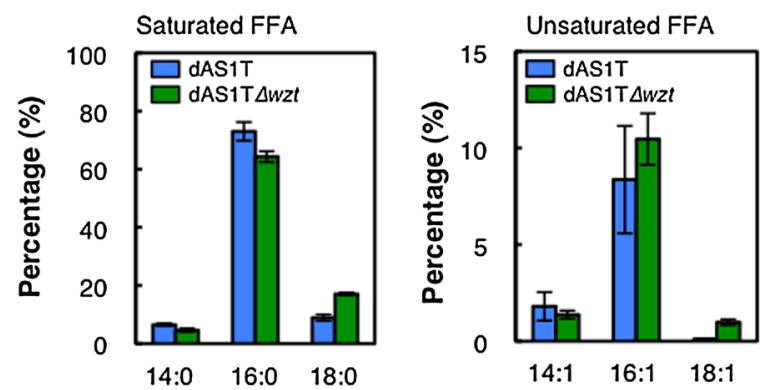

Fig. 5 Cellular and extracellular FFA profiles of the FFA-producing strains. dAS1T and dAS1T $\triangle$ wzt cells were grown under the high-light conditions $\left(180 \mu \mathrm{E} \mathrm{m}^{-2} \mathrm{~s}^{-1}\right)$ in nitrate-containing medium for $192 \mathrm{~h}$. a Cellular and $\mathbf{b}$ extracellular FFA compositions were analyzed by LCMS. Left panel saturated FFA (14:0, 16:0 and 18:0); right panel unsaturated FFA (14:1, 16:1, 18:1). Blue bars dAS1T; green bars dAS1T $\triangle$ wzt. Data shown are the mean \pm SE from biological triplicates

\section{O-antigen deficiency results in protection of photosystems} Time courses of the changes of pigment contents and photosynthetic activities of the cells of WT, dAS1T, and dAS1T $\Delta w z t$ are shown in Fig. 6. The chlorophyll content of WT cells was increased by $50 \%$ during the logarithmic phase of growth and then remained at a high level. The chlorophyll content of dAS1T cells corresponded to 35-55\% of the WT level during cell growth, and was rapidly decreased during the stationary phase, resulting in complete loss of the pigment in $240 \mathrm{~h}$ of cultivation. The chlorophyll content of the dAS1T $\Delta w z t$ cells was higher than that of the dAS1T cells during the logarithmic phase of growth, suggesting that inactivation of $w z t$ alleviated the adverse effects of FFA production on the photosystems. Unlike the content of chlorophyll, the content of phycobiliproteins in $\mathrm{dAS} 1 \mathrm{~T}$ and $\mathrm{dAS} 1 \mathrm{~T} \Delta w z t$ cells was much higher than the wild-type level.

Since the cellular chlorophyll content of the FFAproducing strains greatly changed during the cultivation, the photosynthetic activity was expressed not on a per-chlorophyll basis, but a per-cell basis. (Fig. 6c, d). In each of the strains, the rate of $\mathrm{CO}_{2}$-dependent $\mathrm{O}_{2}$
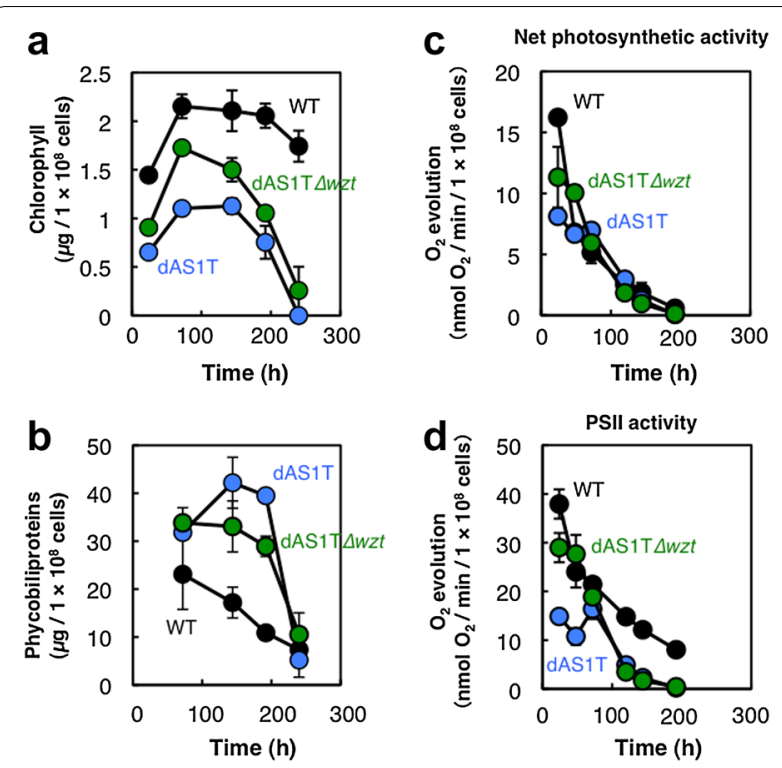

Fig. 6 Changes in the cellular pigment contents, and photosynthetic activities during cultivation. WT, dAS1T, and dAS1T $\triangle$ wzt cells were grown under high light $\left(180 \mu \mathrm{E} \mathrm{m}^{-2} \mathrm{~s}^{-1}\right)$ in nitrate-containing medium. a, b Chlorophyll and phycobiliprotein contents, respectively. Data are the mean \pm SE from the same biological triplicates shown in Fig. 4. c, d Activities of photosynthetic $\mathrm{O}_{2}$ evolution measured with $\mathrm{CO}_{2}$ and $1 \mathrm{mM}$ 1,4-benzoquinone as the electron acceptor, respectively, under illumination at $1000 \mu \mathrm{E} \mathrm{m}^{-2} \mathrm{~s}^{-1}$. Data shown are the mean \pm SE from biological triplicates. Black circles WT; blue circles dAS1T; green circles dAS1TAWZt

evolution, which represents the overall activity of photosynthetic $\mathrm{CO}_{2}$ fixation, was highest in the beginning of cultivation and declined to an insignificant level in $192 \mathrm{~h}$ (Fig. 6c). The activities in the WT and the FFA-producing strains were similar in the late-logarithmic to stationary phase of growth, but in the beginning of cultivation WT showed the highest activity and the dAS1T showed the lowest activity. The PSII electron transport activity of WT, which was measured as the rate of $\mathrm{O}_{2}$ evolution using 1,4-benzoquinone as the electron acceptor, also declined from a high level in the beginning to a low level during the course of cultivation, but unlike the $\mathrm{CO}_{2}$ fixation activity about $20 \%$ of the initial activity remained in the cell even after 192 h of cultivation (Fig. 6d). By contrast, the PSII activity of dAS1T was low from the beginning and sharply declined to a negligible level in $192 \mathrm{~h}$. The PSII activity of dAS1T $\Delta w z t$ cells was twice as high as that of the dAS1T cells in the earlier stage of growth but decreased sharply as in dAS1T to a negligible level in $192 \mathrm{~h}$. These results showed that inactivation of the $w z t$ gene mitigated the adverse effects of FFA production in the dAS1T cells.

Figure 7 shows the effects of growth irradiance on the photosynthetic yield of PSII as determined by measuring 


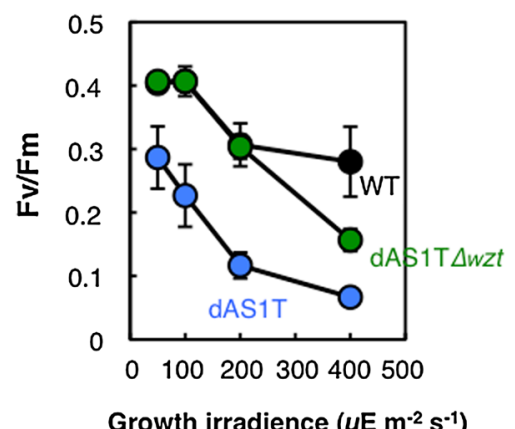

Growth irradience $\left(\mu \mathrm{E} \mathrm{m} \mathrm{m}^{-2} \mathrm{~s}^{-1}\right)$

Fig. 7 Effects of growth irradiance on the photosynthetic yield of WT and the FFA-producing strains. WT, dAS1T, and dAS1T $\triangle$ wzt cells grown under low light $\left(50 \mu \mathrm{E} \mathrm{m}^{-2} \mathrm{~s}^{-1}\right)$ in nitrate-containing medium were transferred to new nitrate-containing medium and cultivated for $36 \mathrm{~h}$ under continuous illumination at the designated light intensities, provided by white-light emitting diode lamps (VBL-SL150-LL, Valore). Black circles WT; blue circles dAS1T; green circles dAS1TAwzt. Data shown are the mean \pm SE from biological triplicates

the $\mathrm{Fv} / \mathrm{Fm}$ ratio. The $\mathrm{Fv} / \mathrm{Fm}$ ratio of $\mathrm{dAS} 1 \mathrm{~T}$ was lower than that of WT even when grown under low-light con-

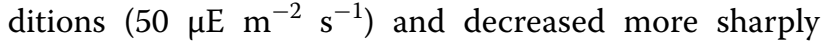
than that of WT as the light intensity during growth was increased (Fig. 7). The Fv/Fm ratio of dAS1T $\Delta w z t$ was essentially the same as that of WT up to a growth irradiance of $200 \mu \mathrm{E} \mathrm{m} \mathrm{m}^{-2} \mathrm{~s}^{-1}$. At $400 \mu \mathrm{E} \mathrm{m} \mathrm{m}^{-2} \mathrm{~s}^{-1}, \mathrm{Fv} /$ Fm declined to a level lower than that of WT, but was much higher than that of dAS1T. Thus, the inactivation of the $w z t$ gene was effective in protecting PSII from photoinhibition.

\section{Discussion}

Among the FFA-producing cyanobacterial mutants constructed to date, the SD277 strain of Synechocystis sp. PCC 6803 is the most productive. Final FFA concentration of about $200 \mathrm{mg} \mathrm{L}^{-1}$ in the external medium was attained with an average secretion rate of $0.44 \mathrm{mg} \mathrm{L}^{-1} \mathrm{~h}^{-1}$ [1]. Synechococcus elongatus PCC 7942 mutants engineered for FFA production have been much less productive (summarized in Ruffing [2]), but the dAS1T mutant constructed in this study shows a relatively high rate of FFA excretion $\left(0.35 \mathrm{mg} \mathrm{L}^{-1} \mathrm{~h}^{-1}\right)$ when cultivated under high-intensity light (Figs. 2, 4). The final FFA concentration in the medium, however, was less than half of that attained by SD277, because of the early death of the dAS1T cells (Figs. 2, 4). Since elimination of the O-antigen layer from the cell surface resulted in a small decrease and increase in cellular and extracellular FFA, respectively, and significantly enhanced cell growth (Fig. 4), it is deduced that accumulation of excessive FFA in the cell was the cause of the early death of dAS1T. Unlike the SD277 mutant of Synechocystis sp. PCC 6803, which excretes about $95 \%$ of the total FFA into the medium [1], the dAS1T cells carry about $85 \%$ of the total FFA in the culture (Fig. 4B). These results indicate that the FFA productivity of the S. elongatus mutant is limited by the slow permeation of FFA across the cell envelope.

Taking into account the FFAs associated with in the cells, the total FFA contents in the cultures of the SD277 mutant of Synechocystis sp. PCC 6803 and the $\Delta w z t$ derivative of $\mathrm{dAS} 1 \mathrm{~T}$ are calculated to be 210 and $650 \mathrm{mg} \mathrm{L}^{-1}$, respectively, at the end of cultivation. Thus, S. elongatus PCC 7942 has a much greater capacity for fatty acid biosynthesis than Synechocystis sp. PCC 6803. The average rate of total FFA production by the S. elongatus PCC 7942 mutant is calculated to be $2.7 \mathrm{mg} \mathrm{L}^{-1} \mathrm{~h}^{-1}$, which is comparable to the rates of TAG production reported for the green algae, Chlorella vulgaris, Chlorella emersonii, and Nannochloropsis gaditana $[15,16]$. Since most cyanobacteria including S. elongatus PCC 7942 accumulate glycogen as the storage material under the conditions of $\mathrm{N}$ limitation [17-19], it has been supposed that cyanobacteria are not well suited for lipid production. The present results, however, demonstrate that certain species of cyanobacteria have a large potential as a source of lipids to be used as biodiesel.

Although inactivation of the $w z t$ gene in dAS1T increased the FFA secretion rate to $0.41 \mathrm{mg}^{-1} \mathrm{~L}^{-1}$ and delayed accumulation of FFAs in the cell (Fig. 4), the cellular FFA content of the $\Delta w z t$ strain was increased to a level as high as that in the parental strain after $240 \mathrm{~h}$ of cultivation. The final concentration of FFA in the external medium was about $100 \mathrm{mg} \mathrm{L}^{-1}$, being about half of that attained by the Synechocystis SD277 mutant in $450 \mathrm{~h}$. The cultures of the $\Delta w z t$ strain were green at $t=240 \mathrm{~h}$ (Fig. 4A), but assumed a blue coloration after $264 \mathrm{~h}$ of cultivation (not shown), indicating that the early-death phenotype of the dAS1T strain was only partially rescued by the inactivation of the wzt gene. To further increase the productivity of the extracellular FFA, the rate of FFA excretion needs to be increased. To this end, the RND-type export system having the capacity of FFA excretion, which was recently identified in S. elongatus PCC 7942 [7], would be useful. Given the large capacity for FFA production found in the S. elongatus 7942 mutants, it would also be necessary to introduce multiple export systems for FFAs, including those identified in other bacterial species [20-22]. Given the presence of the intracellular membrane system (the thylakoids) in the cyanobacterial cell, correct targeting of the FFA exporters to the cytoplasmic membrane would be critical particularly in the attempts to overexpress the transporters. The other possible strategy to increase the FFA in the external medium is to regulate FFA production in the cell during the stationary phase of growth. Without 
cell division, accumulation of FFA to a lethal level for the cell is inevitable if the rate of FFA production exceeds the rate of its excretion. This approach will sacrifice the large capacity of FFA production in the S. elongatus cell, but by balancing the rates of FFA production and excretion, sustained excretion of FFA for longer periods of time will be possible. Upon successful functional expression of FFA exporter(s), FFA production can be deregulated to meet the increased rate of FFA excretion.

Using an aas single mutant of S. elongatus PCC 7942 (dAS1), Takatani et al. [10] showed that deacylation of membrane lipids is activated under high-light conditions [10]. PSII is unstable in dAS1 and is much more sensitive to high light than in WT, presumably due to accumulation of FFAs and/or lysolipids [10]. As in dAS1, the PSII activity of dAS1T is hypersensitive to high light, but elimination of the $\mathrm{O}$-antigen layer effectively protects PSII from photoinhibition (Fig. 7), suggesting that intracellular accumulation of FFA is the major cause of destabilization of PSII. FFAs act as surfactants and are known to have various adverse effects on cellular activities [23]. dAS1T produces FFAs not only from membrane lipids but from acyl-ACP via the action of the "TesA thioesterase. Since the high-light conditions stimulate photosynthesis and the synthesis of acyl-ACP, production of FFAs from acyl-ACP would also be stimulated under high-light conditions. dAS1T is therefore supposed to be suffering more seriously than dAS1 from various biochemical and biophysical defects including photoinhibition of PSII under the high-light conditions. It may be hence not surprising that dAS1T cells die under the high-light conditions in an unusual manner, i.e., with complete degradation of chlorophyll but not of phycobiliproteins, giving rise to the blue coloration of the culture (Figs. 2b, 4Ab). Although the inactivation of the wzt gene did not fully rescue the early-death phenotype of dAS1T, enhancement of cell growth, and FFA production, and protection of the activity of PSII in dAS1T $\Delta w z t$ suggest that the O-antigen deficiency effectively decreased intracellular accumulation of FFAs. Simkovsky et al. [13] suggested that $S$. elongatus mutants defective in O-antigen production are suited for biofuel production, as they are resistant to grazing by amoebae and capable of autoflocculation without showing apparent growth defects [13]. The present results show that the loss of $\mathrm{O}$-antigen brings about additional useful traits in the FFA-producing $S$. elongatus strains: facilitation of FFA release from the cells, alleviation of photoinhibition, and enhancement of growth and FFA production. Thus, all the known phenotypes resulting from $\mathrm{O}$-antigen deficiency would directly contribute to the productivity, making the mutations in $\mathrm{O}$-antigen production indispensable for the construction of an efficient FFA-producing system.

\section{Conclusions}

Synechococcus elongatus PCC 7942 has an unusually large capacity to synthesize fatty acids, which is to be exploited for biofuel production. Currently, the high activity of FFA synthesis results in intracellular accumulation of FFA, which adversely affects cellular metabolism and eventually kills the cells. To achieve high rate production of FFA, excretion of FFA out of the cells via both passive and positive mechanisms would be required. Inactivation of the wzt gene is effective in facilitating FFA secretion and will be essential.

\section{Methods}

\section{Strains and growth conditions}

Strains and plasmids used in this study are shown in Additional file 1: Table S1. A derivative of Synechococcus elongatus PCC 7942 that is cured of the resident small plasmid pUH24 (strain SPc, hereafter designated simply as wild-type strain (WT) [24]) and the FFA-producing mutants were grown photoautotrophically at $30{ }^{\circ} \mathrm{C}$ under continuous illumination, which was provided by fluorescent lamps unless otherwise stated, with aeration by air supplemented with $2 \%(v / v) \mathrm{CO}_{2}$. The basal medium used was a nitrogen-free derivative of the BG11 medium described previously [25], which was supplemented with $15 \mathrm{mM} \mathrm{KNO}_{3}$ and $3.75 \mathrm{mM}\left(\mathrm{NH}_{4}\right)_{2} \mathrm{SO}_{4}$ as a nitrogen source to prepare nitrate-containing medium and ammonium-containing medium, respectively. The media were buffered with $20 \mathrm{mM}$ HEPES-KOH (pH 8.2). When appropriate, spectinomycin was added to the medium at $15 \mu \mathrm{g} \mathrm{ml}^{-1}$. Since the PSII activity of the FFA-producing strains declines sharply during stationary phase of growth, exponentially growing cells $\left(\mathrm{OD}_{730}=0.5-1.0\right)$ were used to inoculate new cultures to be used for all the experiments. Optical density of the cultures was measured at $730 \mathrm{~nm}$ using a spectrophotometer (UV-1700, Shimadzu). Cell volume and cell number were determined using a particle counter/analyzer (CDA-1000, Sysmex).

\section{Construction of the FFA-producing strains}

Transformation of cyanobacteria was performed as described by Williams and Szalay [26]. The FFA-producing mutant (designated dAS1T) was obtained by introduction of a truncated 'tes $A$ gene from $E$. coli transcriptionally fused to nirA promoter $\left(P_{\text {nirA }}\right)$ from $S$. elongatus into the aas-deficient mutant dAS1sacB (Fig. S1 in Takatani et al. [10]). The plasmid used to introduce the

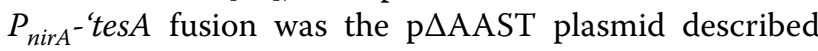
previously [7]. dAS1sacB was transformed with $\mathrm{p} \triangle \mathrm{AAST}$ and the mutant dAS1T was obtained by counterselection of SacB in the presence of $10 \%$ sucrose [27]. Segregation of the alleles was confirmed by PCR analysis of 
the genomic DNA from selected transformants using the primers 0918Sf and 0918Sr (Additional file 2: Table S2).

\section{Inactivation of the wzt gene}

For construction of the $w z t$-deficient mutant derived from dAS1T (dAS1T $\Delta w z t$ ), a 945-bp DNA fragment carrying the first 45 bases of the wzt ORF and 900 bp of its $5^{\prime}$ flanking sequence was amplified by PCR using the primers 1244Uf carrying an added EcoRI site and 1244Ur carrying an added BamHI site. 1827 bp of the $3^{\prime}$ flanking region of $w z t$ was also amplified by PCR using the primers 1244Df carrying an added Xbal site and 1244Dr carrying an added HindIII site (Additional file 2: Table S2). These DNA fragments were integrated sequentially between the EcoRI and BamHI sites and the XbaI and HindIII sites of the pUC19 vector, respectively, to yield the plasmid $\mathrm{p} \triangle \mathrm{WZT}$. The spectinomycin resistance cassette derived from pRL463 [28] was cloned into the BamHI site of $\mathrm{p} \Delta \mathrm{WZT}$ to yield the plasmid $\mathrm{p} \Delta \mathrm{WZTSpe}^{\mathrm{r}}$. dAS1T cells were transformed with $\mathrm{p} \Delta \mathrm{WZTSpe}^{\mathrm{r}}$ to construct the $\mathrm{dAS1T} \Delta w z t$ strain via replacement of most of the $w z t$ ORF with the spectinomycin resistance cassette. Successful genome segregation was confirmed by PCR using the primers 1244Sf and 1244Sr (Additional file 2: Table S2).

\section{FFA analysis}

For analysis of cellular and extracellular FFA, 5-30 ml aliquots of the cultures were centrifuged at $1700 \mathrm{~g}$ for $15 \mathrm{~min}$ to separate the cells and the medium. The supernatant was transferred to CryoTubes (Thermo Fisher Scientific) and the cells were resuspended in $1 \mathrm{~mL}$ of methanol. The samples were stored at $-20{ }^{\circ} \mathrm{C}$ until use. For enzymatic determination of the total concentration of FFA in the medium, the supernatant was analyzed using the Free Fatty Acid Quantification Kit (BioVision) according to the manufacture's instruction. For analysis of the cellular and extracellular FFA profiles and the total FFA content in the cells, samples were supplemented with the internal standards (16:0[d3] and 18:0[d3]), extracted with a modified Folch method [29, 30], and analyzed by LC-MS [10, 30]. To obtain the standard curves for FFAs, methanolic solutions of FFAs were treated exactly as the samples were, including the step of extraction with the modified Folch method, and subjected to LC-MS analysis.

\section{Determination of $\mathrm{Chl}$ and phycobiliproteins}

Chlorophyll (Chl) was determined as described by Mackinney [31]. For the measurement of phycobiliproteins, cells were disrupted as described by Aoki et al. [32]. The concentration of phycobiliproteins (phycocyanin and allophycocyanin) in the supernatant was determined from the absorbance at 620 and $650 \mathrm{~nm}$, using the equation described by Tandeau de Marsac and Houmard [33].
$\mathrm{Chl}$ and $\mathrm{PC}$ contents were normalized to the cell number corresponding to $1.0 \times 10^{8}$ of the cells.

\section{Measurement of photosynthetic activity}

Photosynthetic oxygen-evolving activity of the cyanobacterial cultures was measured at $30^{\circ} \mathrm{C}$ under illumination at $1000 \mu \mathrm{mol}$ photons $\mathrm{m}^{-2} \mathrm{~s}^{-1}$, using a Clark-type oxygen electrode (DW1, Hansatech). The photosynthetic yield of PSII was measured using an AquaPen-C fluorometer (AP-C100, Photon Systems Instruments).

\section{Preparation of RNA and semi-quantitative RT-PCR}

Total RNA was extracted from dAS1T cells grown with either nitrate or ammonium under high light $\left(180 \mu \mathrm{E} \mathrm{m}^{-2} \mathrm{~s}^{-1}\right)$ at $t=48 \mathrm{~h}$, using a combination of the TRIzol $^{\circledR}$ Reagent (Life technologies) and the SV total RNA isolation system (Promega) as described by Kato et al. [7]. The isolated total RNA $(1 \mu \mathrm{g})$ was used for the synthesis of cDNA, using a SuperScript ${ }^{\mathrm{TM}}$ III First-Strand Synthesis System (Life Technologies) and random primers according to the manufacture's instruction. The obtained cDNA was used as the template for PCR analysis using the primers specific to tes $A$ and $r n p B$ (Additional file 3: Table S3).

\section{Additional files}

Additional file 1: Table S1. Strains and plasmids used in this study Relevant characteristics of strains and plasmids are described.

Additional file 2: Table S2. Oligonucleotides used for the construction of the mutants. Sequences are given from the $5^{\prime}$ end to the $3^{\prime}$ end.

Additional file 3: Table S3. Oligonucleotides used for semi-quantitative RT-PCR. Sequences are given from the $5^{\prime}$ end to the $3^{\prime}$ end.

\section{Abbreviations}

FFAs: free fatty acids; TAG: triacylglycerol; Aas: acyl-ACP synthetase; ACP: acyl-acyl carrier protein; LPS: lipopolysaccharide; Sper: spectinomycin resistance; WT: wild type; SPc: small-plasmid-cured; ORF: open reading frame; Chl: chlorophyll; RT-PCR: reverse transcription-PCR.

\section{Authors' contributions}

AK designed the experiments, carried out them, and participated in data analysis and in drafting the manuscript. KU constructed the recombinant strains and helped to analyze the data. NT designed the experiments and participated in drafting the manuscript. KI carried out FFA analysis using LC-MS and analyzed the data. MM performed semi-quantitative RT-PCR analysis of 'tesA transcript. KK, MA, and SM helped to design the experiments and analyze the data. TO conceived the study, participated in its design and coordination, and helped to draft the manuscript. All authors read and approved the manuscript.

\section{Author details}

${ }^{1}$ Graduate School of Bioagricultural Sciences, Nagoya University,

Nagoya 464-8601, Japan. ${ }^{2}$ Institute for Advanced Biosciences, Keio University, Tsuruoka, Yamagata 997-0052, Japan. ${ }^{3}$ Department of Biological Chemistry, Chubu University, Kasugai 487-8501, Japan. ${ }^{4}$ Japan Science and Technology Agency, CREST, Tokyo, Japan. ${ }^{5}$ Present Address: Laboratory for Metabolomics, RIKEN Center for Integrative Medical Sciences, Yokohama 230-0045, Japan.

${ }^{6}$ Laboratory of Molecular Plant Physiology, Graduate School of Bioagricultural Sciences, Nagoya University, Furo, Chikusa, Nagoya 464-8601, Japan. 


\section{Acknowledgements}

This study was supported by the Japan Science and Technology Agency CREST funding program in the area of 'Creation of Basic Technology for Improved Bioenergy Production through Functional Analysis and Regulation of Algae and Other Aquatic Microorganisms'.

\section{Competing interests}

The authors declare that they have no competing interests.

Received: 2 February 2016 Accepted: 12 April 2016

Published online: 23 April 2016

\section{References}

1. Liu X, Sheng J, Curtiss R. Fatty acid production in genetically modified cyanobacteria. Proc Natl Acad Sci USA. 2011;108:6899-904

2. Ruffing AM. Improved free fatty acid production in cyanobacteria with Synechococcus sp. PCC 7002 as host. Front Bioeng Biotechnol. 2014;2:17

3. Ruffing AM, Jones HD. Physiological effects of free fatty acid production in genetically engineered Synechococcus elongatus PCC 7942. Biotechnol Bioeng. 2012;109:2190-9.

4. Hu Q, Sommerfeld M, Jarvis E, Ghirardi M, Posewitz M, Seibert M, et al. Microalgal triacylglycerols as feedstocks for biofuel production: perspectives and advances. Plant J. 2008;54:621-39.

5. Kaczmarzyk D, Fulda M. Fatty acid activation in cyanobacteria mediated by acyl-acyl carrier protein synthetase enables fatty acid recycling. Plant Physiol. 2010;152:1598-610.

6. Ruffing A. Borrowing genes from Chlamydomonas reinhardtii for free fatty acid production in engineered cyanobacteria. J Appl Phycol. 2013:25:1495-507.

7. Kato A, Takatani N, Use K, Uesaka K, Ikeda K, Chang Y, et al. Identification of a cyanobacterial RND-type efflux system involved in export of free fatty acids. Plant Cell Physiol. 2015;56:2467-77.

8. Maeda S, Kawaguchi Y, Ohe TA, Omata T. cis-acting sequences required for NtcB-dependent, nitrite-responsive positive regulation of the nitrate assimilation operon in the cyanobacterium Synechococcus sp. strain PCC 7942. J Bacteriol. 1998;180:4080-8.

9. Aichi M, Maeda S, Ichikawa K, Omata T. Nitrite-responsive activation of the nitrate assimilation operon in Cyanobacteria plays an essential role in up-regulation of nitrate assimilation activities under nitrate-limited growth conditions. J Bacteriol. 2004;186:3224-9.

10. Takatani N, Use K, Kato A, Ikeda K, Kojima K, Aichi M, et al. Essential role of acyl-ACP synthetase in acclimation of the cyanobacterium Synechococcus elongatus strain PCC 7942 to high-light conditions. Plant Cell Physiol. 2015;56:1608-15.

11. Sheu CW, Freese E. Lipopolysaccharide layer protection of gramnegative bacteria against inhibition by long-chain fatty acids. J Bacteriol. 1973;115:869-75.

12. Schielke S, Schmitt C, Spatz C, Frosch M, Schubert-Unkmeir A, Kurzai O. The transcriptional repressor FarR is not involved in meningococcal fatty acid resistance mediated by the FarAB efflux pump and dependent on lipopolysaccharide structure. Appl Environ Microbiol. 2010;76:3160-9.

13. Simkovsky R, Daniels EF, Tang K, Huynh SC, Golden SS, Brahamsha B. Impairment of $\mathrm{O}$-antigen production confers resistance to grazing in a model amoeba-cyanobacterium predator-prey system. Proc Natl Acad Sci USA. 2012;109:16678-83.

14. Ho SH, Nakanishi A, Ye X, Chang JS, Hara K, Hasunuma T, et al. Optimizing biodiesel production in marine Chlamydomonas sp. JSC4 through metabolic profiling and an innovative salinity-gradient strategy. Biotechnol Biofuels. 2014;7:97.
15. Talebi A, Mohtashami S, Tabatabaei M, Tohidfar M, Bagheri A, Zeinalabedini $M$, et al. Fatty acids profiling: a selective criterion for screening microalgae strains for biodiesel production. Algal Res. 2013;2:258-67.

16. San Pedro A, Gonzalez-Lopez C, Acien F, Molina-Grima E. Marine microalgae selection and culture conditions optimization for biodiesel production. Bioresour Technol. 2013;134:353-61.

17. Görl M, Sauer J, Baier T, Forchhammer K. Nitrogen-starvation-induced chlorosis in Synechococcus PCC 7942: adaptation to long-term survival. Microbiology. 1998;144:2449-58.

18. Osanai T, Imamura S, Asayama M, Shirai M, Suzuki I, Murata N, et al. Nitrogen induction of sugar catabolic gene expression in Synechocystis sp. PCC 6803. DNA Res. 2006;13:185-95.

19. Hasunuma T, Kikuyama F, Matsuda M, Aikawa S, Izumi Y, Kondo A. Dynamic metabolic profiling of cyanobacterial glycogen biosynthesis under conditions of nitrate depletion. J Exp Bot. 2013;64:2943-54.

20. Lennen RM, Politz MG, Kruziki MA, Pfleger BF. Identification of transport proteins involved in free fatty acid efflux in Escherichia coli. J Bacteriol. 2013;195:135-44.

21. Lee EH, Shafer WM. The farAB-encoded efflux pump mediates resistance of gonococci to long-chained antibacterial fatty acids. Mol Microbiol. 1999;33:839-45.

22. Alnaseri H, Arsic B, Schneider J, Kaiser J, Scinocca Z, Heinrichs D, et al. Inducible expression of a resistance-nodulation-division-type efflux pump in Staphylococcus aureus provides resistance to linoleic and arachidonic acids. J Bacteriol. 2015;197:1893-905.

23. Desbois AP, Smith VJ. Antibacterial free fatty acids: activities, mechanisms of action and biotechnological potential. Appl Microbiol Biotechnol. 2010;85:1629-42.

24. Kuhlemeier CJ, Thomas AA, van der Ende A, van Leen RW, Borrias WE, van den Hondel CA, et al. A host-vector system for gene cloning in the cyanobacterium Anacystis nidulans R2. Plasmid. 1983;10:156-63.

25. Suzuki I, Kikuchi H, Nakanishi S, Fujita Y, Sugiyama T, Omata T. A novel nitrite reductase gene from the cyanobacterium Plectonema boryanum. J Bacteriol. 1995;177:6137-43.

26. Williams JG, Szalay AA. Stable integration of foreign DNA into the chromosome of the cyanobacterium Synechococcus R2. Gene. 1983;24:37-51.

27. Cai YP, Wolk CP. Use of a conditionally lethal gene in Anabaena sp. strain PCC 7120 to select for double recombinants and to entrap insertion sequences. J Bacteriol. 1990;172:3138-45.

28. Elhai J, Wolk C. Conjugal transfer of DNA to cyanobacteria. Methods Enzymol. 1988;167:747-54.

29. Folch J, Lees M, Sloane Stanley GH. A simple method for the isolation and purification of total lipides from animal tissues. J Biol Chem. 1957;226:497-509.

30. Ikeda K. Mass-spectrometric analysis of phospholipids by target discovery approach. In: Yokomizo T, Murakami M, editors. Bioactive lipid mediators. Japan: Springer; 2015. p. 349-56.

31. Mackinney G. Absorption of light by chlorophyll solutions. J Biol Chem. 1941;140:315-22

32. Aoki R, Goto T, Fujita Y. A heme oxygenase isoform is essential for aerobic growth in the cyanobacterium Synechocystis sp. PCC 6803: modes of differential operation of two isoforms/enzymes to adapt to low oxygen environments in cyanobacteria. Plant Cell Physiol. 2011;52:1744-56.

33. de Marsac NT, Houmard J. Complementary chromatic adaptationphysiological conditions and action spectra. Methods Enzymol. 1988;167:318-28. 